\title{
Examining Dislocation Structures in Mo Pre-Strained Fibers via Electron Tomography
}

V. McCreary, ${ }^{1}$ G.S. Liu, ${ }^{1}$ J. Kwon, ${ }^{2}$ P. Sudharshan Phani, ${ }^{3}$ K. E. Johanns, ${ }^{3}$ I.M. Robertson, ${ }^{1}$ M.J. Mills, ${ }^{2}$ G.M. Pharr, ${ }^{3,4}$ E. P. George. ${ }^{3,4}$

${ }^{1}$ Dept. of Materials Science and Engineering, University of Illinois, Urbana, IL 61801.

${ }^{2}$ Dept. of Materials Science and Engineering, Ohio State University, Columbus, OH 43210.

${ }^{3}$ Dept. of Materials Science and Engineering, University of Tennessee, Knoxville, TN 37996.

${ }^{4}$ Mat. Sci. \& Tech. Div., Oak Ridge National Laboratory, Oak Ridge, TN 37831.

Mo-alloy fibers, Mo-10Al-4Ni at.\%, (henceforth, Mo fibers) with diameters in the range $300-550 \mathrm{~nm}$ were grown embedded in a NiAl matrix. Samples of this composite were compressed to 4 and $16 \%$ engineering strain. To determine the deformed microstructure in the fibers, the NiAl was etched to expose 20-40 $\mu \mathrm{m}$-long Mo fibers which were extracted and collected on a copper holey-carbon TEM grid. Conventional electron microscopy has been employed to show that the density of dislocations increases and the distribution, at least in projection, becomes more uniform with increasing strain [1]. The dislocations were found to have a Burger vector of the type $a / 2<111>$. What is lacking from our knowledge of the dislocation structure is how the dislocations are spatially distributed throughout the fiber. To determine this distribution, images for electron tomography were acquired using conventional diffraction contrast imaging conditions as well as scanning transmission electron microscopy.

Scanning transmission electron microscopy images were taken with a Tecnai F20 FEG system operating at $200 \mathrm{kV}$. Fig. 1 shows the dual-beam FIB extraction of a $16 \%$ prestrained Mo fiber which is then attached to a molybdenum Omniprobe TEM grid. A representative bright-field diffraction contrast image from a STEM tilt series is shown in Fig. 2. Obtaining diffraction contrast using STEM imaging has been discussed recently in [2]. Conventional transmission electron microscopy bright-field contrast images, Fig. 3, were taken of the Mo fibers still on the copper TEM grid. These were taken with a JEOL $2010 \mathrm{LaB} 6$ operating at $200 \mathrm{kV}$. The methods used to construct tomograms from these tilt series are detailed elsewhere [3].

From the images presented in Fig. 3, it is evident that the dislocations do not simply extend across the fiber but show evidence of cross-slip - examples of this can be seen in Fig. 4. This cross-slip is presumably a consequence of the dislocations encountering and bypassing defects. Possible obstacles such as small dislocation loops $<30 \mathrm{~nm}$ in diameter are seen in the images presented in Fig. 3. Dislocation half-loops were also observed to extend out from the edge of the fiber, although the point of origin could not be discerned from these tomograms. The complexity of dislocation tangles along with the presence of small prismatic loops, especially in the $16 \%$-strained fibers, posed a challenge in fully characterizing the dislocation distribution and microstructure without three-dimensional information. The tomograms and dislocation models have yielded much needed insight as to how the dislocations are distributed throughout the fiber and 
this information will provide input and checks for dislocation dynamics simulations of deformation of these fibers [4].

\section{References}

[1] P. Sudharshan Phani et al., Acta Mater. 59 (2011) 2172.

[2] P.J. Phillips, M.J. Mills, and M. De Graef, Systematic Row and Zone Axis STEM Defect Image Simulations. Phil. Mag. A, in press (2011).

[3] G.S. Liu, I.M. Robertson, J. Mater. Res., 26 (2011).

[4] This work was sponsored by the Center for Defect Physics, an Energy Frontier Research Center supported by the U.S. Department of Energy, Office of Basic Energy Sciences.
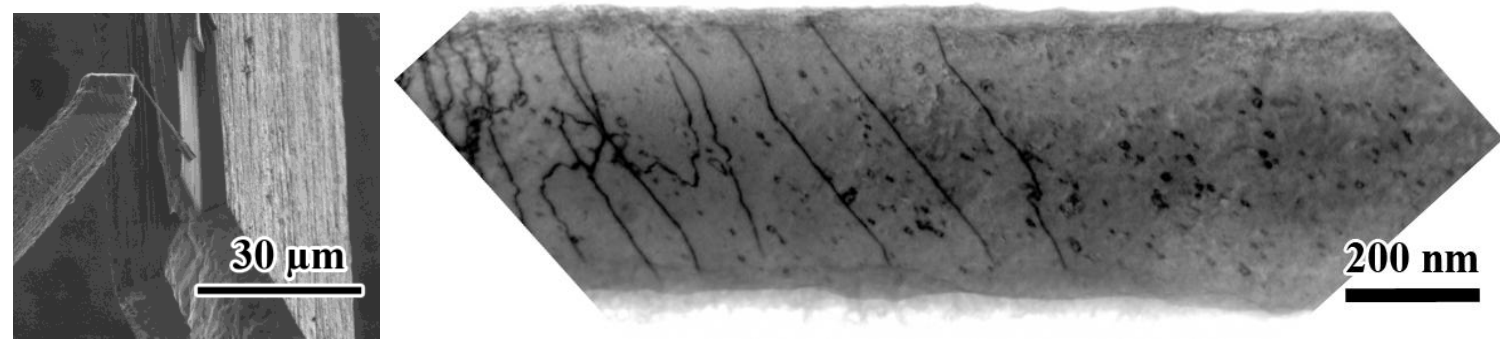

FIG 1. (left) Focused ion beam extraction of a Mo fiber. The fiber is then attached to an Omniprobe TEM grid.

FIG 2. (right) Bright-field STEM image of $16 \%$ pre-strained Mo fiber. From a tilt series taken approximately every $1.5^{\circ}$ along a $<110>$ Kikuchi band over a range of approximately $65^{\circ}$.
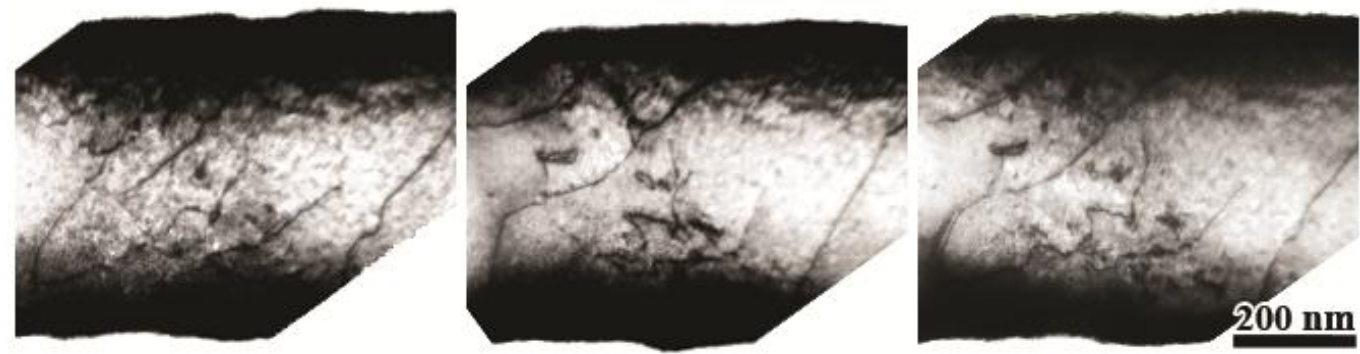

FIG 3. Aligned bright-field TEM images of $16 \%$ pre-strained Mo fiber from a tilt series taken every $2^{\circ}$ over a range of $57^{\circ}$. Example images are tilted $\pm 18^{\circ}$ about $0.6^{\circ}$.
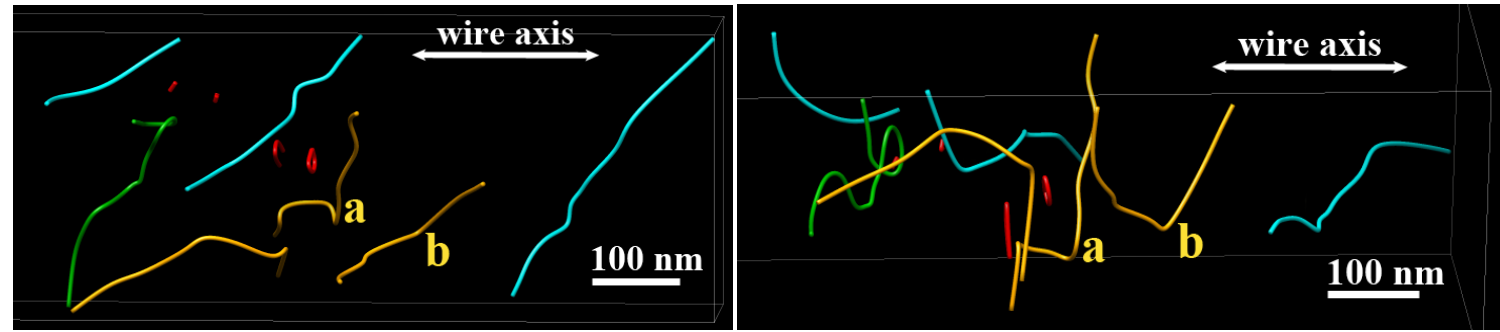

FIG 4. Two views of a traced dislocation model constructed with the tilt series presented in Fig. 3. Note cross-slip in dislocations near markers ' $a$ ' and ' $b$.' 